\title{
The Urinary Albumin-to-Creatinine Ratio Is a Potential Predictor of Target Lesion Revascularization After Percutaneous Coronary Intervention With Coronary Stents
}

\author{
Takuya OKada, ${ }^{1}$ MD, Masaru Yuge, ${ }^{2}$ MD, Takeo Kawaguchi, ${ }^{2}$ MD, and Yukihiro Hojo, ${ }^{3}$ MD
}

\begin{abstract}
SUMMARY
The association between the urinary albumin-to-creatinine ratio (UACR) and target lesion revascularization (TLR) is unknown in patients who are implanted with drug-eluting stents (DESs) or bare metal stents (BMSs) for the treatment of coronary artery disease. Of 231 Japanese patients who were implanted with DESs and/or BMSs during percutaneous coronary intervention (PCI) between July 2009 and January 2011, 118 underwent follow-up coronary angiography at 6 to 9 months after PCI; 103 were negative for qualitative tests for urine protein: 32 (31.0\%)/103 patients underwent TLR for severe in-stent restenosis (ISR) and 71 did not. On the next day after admission to the hospital, first-morning-void spot urine samples were collected to calculate UACR based on urinalysis results. Pearson's product-moment correlation coefficients indicated positive associations of UACR with late loss as assessed by quantitative coronary analysis in the overall cohort, $(r=+0.515, P<0.0001)$, the DES subgroup $(r=+0.443, P<0.0001)$, and the BMS subgroup $(r=$ $+0.652, P<0.0001)$. The incidence of multivessel lesions was significantly higher $(P<0.05)$ in the TLR group. UACR was significantly higher $(P<0.01)$ in the TLR group $(23.88 \pm 31.8 \mathrm{mg} / \mathrm{gCr})$ than in the control group $(6.29 \pm 7.46 \mathrm{mg} /$ $\mathrm{gCr}$ ). Multivariate logistic regression analysis revealed UACR (odds ratio: 1.07; 95\% confidence interval: $1.02-1.12 ; P<$ 0.01 ) to be associated with TLR. UACR was suggested to be a potential predictor of TLR required for severe ISR after PCI with coronary stents. (Int Heart J 2014; 55: 409-415)
\end{abstract}

Key words: Microalbuminuria, Drug-eluting stent, Bare metal stent, In-stent restenosis, Late loss

$\mathrm{M}$ icroalbuminuria is a risk factor for cardiovascular and cerebrovascular events and is associated with a poor life prognosis in patients with an adverse cardiovascular risk profile. ${ }^{1-5)}$ Furthermore, low-grade albuminuria with a urinary albumin concentration below the conventional threshold levels for microalbuminuria $(20-200 \mathrm{mg} / \mathrm{gCr}$ for males and $30-300 \mathrm{mg} / \mathrm{gCr}$ for females) is currently recognized as a risk factor for cardiovascular disease (CVD) ${ }^{6,7)}$ In addition, microalbuminuria is independently associated with ischemic electrocardiographic abnormalities. $\left.{ }^{8}\right)$ The Framingham Heart Study analyzed the population of nondiabetic and nonhypertensive individuals and reported threefold increases in CVD risk when the urinary albumin-to-creatinine ratio (UACR) exceeded 3.9 and $7.5 \mathrm{mg} / \mathrm{gCr}$ in males and females, respectively." Microalbuminuria caused by minimal glomerular damage suggests vascular endothelial injury in the glomeruli, which led us to hypothesize the presence of a possible association between arteriosclerosis progression and elevated UACR. Advanced arteriosclerosis, severe calcification, and intense vascular remodeling may predispose stent malexpansion and/or malapposition in patients who underwent percutaneous coronary intervention (PCI) with coronary stents for the treatment of coronary artery disease (CAD) and can provoke in- stent restenosis (ISR) after PCI. Since ISR is a serious complication of coronary stenting that has proven extremely difficult to treat once developed, it is of clinical relevance to make an effort to find a laboratory approach by which ISR can be indirectly predicted by simple procedures that can be performed in routine clinical practice. In the present retrospective, singlecenter, open-label study, we examined the association between microalbuminuria as assessed with UACR and ISR after PCI using coronary stents.

\section{Methods}

Study population: Of 231 Japanese patients with CAD who had been implanted with drug-eluting stents (DESs) or bare metal stents (BMSs) for the treatment of CAD between between July 2009 and January 2011, 118 underwent follow-up coronary angiography (CAG) at 6 to 9 months after PCI between April 2010 and July 2011; 103 in the latter cohort of patients were negative for qualitative tests for urine protein. All patients gave written informed consent to use their clinical and laboratory data in the present study that was approved by the local ethics committee before its conduct in accordance with

From the Departments of ${ }^{1}$ General Medicine and ${ }^{2}$ Cardiology, Odawara Municipal Hospital, Odawara, Kanagawa, and ${ }^{3}$ Department of Cardiology, Jichi Medical University, Shimotsuke, Tochigi, Japan.

Address for correspondence: Takuya Okada, MD, Department of General Medicine, Odawara Municipal Hospital, 46 Kuno, Odawara, Kanagawa 250-8558, Japan. E-mail: takuyaodawara@outlook.com

Received for publication January 21, 2014. Revised and accepted April 6, 2014.

Released in advance online on J-STAGE August 4, 2014.

All rights reserved by the International Heart Journal Association. 
the Declaration of Helsinki.

CAG, PCI, and UACR: During PCI, CAG was performed via the femoral or radial artery approach under local anesthesia. The following stent delivery systems were used: Taxus Express (Boston Scientific, Natick, MA), Taxus Liberte (Boston Scientific), Promus (Boston Scientific), Xience V (Abbott Vascular, Santa Clara, CA), Multi-Link Vision (Abbott Vascular), MultiLink Mini Vision (Abbott Vascular), Endeavor (Medtronic CardioVascular, Santa Rosa, CA), Endeavor Sprint (Medtronic CardioVascular), Driver (Medtronic CardioVascular), and Driver Sprint (Medtronic CardioVascular). The guiding sheath ( $5 \mathrm{Fr}$ ) and the catheter ( $5 \mathrm{Fr}$ ) were inserted into the artery to conduct PCI with balloon-mount delivery system(s) and DESs/ BMSs in the target lesion where a significant stenosis as defined by the American Heart Association classification ${ }^{10)}$ was detected. The type and number of stent(s) to implant were determined at the interventionist's discretion. Heparin (5,000 U) was administered intravenously to the site of stent implantation. Dual antiplatelet therapy using aspirin (100 mg/day) and clopidogrel (75 mg/day), ticlopidine (200 mg/day), or cilostazol (200 mg/day) was conducted for at least 3 months after PCI. Follow-up CAG was performed at 6 to 9 months after PCI.

Blood and first-morning-void spot urine samples were collected with the vacuum blood-collecting tube and the lightresistant conical bottom test tube before breakfast on the next day of PCI. The blood and urine samples collected were centrifuged and stored at $4^{\circ} \mathrm{C}$, with EDTA $2 \mathrm{~K}$ as an anticoagulant for blood and without use of a preservative for urine, for 15 hours prior to analyses. UACR was calculated based on the measured values in routine urinalysis by immunoturbidimetry as described previously. ${ }^{11)}$

Quantitative coronary analysis: Xcelera QCA Software (Xcelera, Philips Medical Systems; Best, North Brabant, The Netherlands) was used to perform quantitative coronary analysis (QCA) for measuring the dimensions (eg, diameter and length) of the target coronary artery before determination of the size of the stent(s) to implant, as well as for assessing the morphology of the target lesion after PCI. Follow-up CAG at 6 to 9 months after PCI was conducted at the same angiographic projection angles as those used when stent(s) were implanted during PCI. Reference vessel diameter, minimum lumen diameter (MLD), stenosis rate, stent length (SL), and stent segment length were measured to calculate acute gain and late loss at the site of stent implantation. Minimum stent diameter was considered as MLD when using multiple stents at the time of ISR assessment. The total length of stents was considered as SL when consecutively implanting multiple stents in the same lesion. Target lesion revascularization (TLR) was conducted when exercise myocardial scintigraphy with 201-thallium indicated tracer redistribution, when the patient showed an obvious exertional chest pain, or when QCA at the time of follow-up $\mathrm{CAG}$ revealed $\mathrm{a} \geq 75 \%$ stenosis.

Statistical analysis: Continuous variables, expressed as the mean $\pm \mathrm{SD}$, were compared among groups according to Student's $t$-test or the Kruskal-Wallis test. Categorical and binary variables, expressed as numerals (percentages), were compared between groups according to Mann-Whitney's $U$-test and Pearson's $\chi^{2}$ test, respectively. Pearson's product-moment correlation coefficient was calculated among variables. Univariate and multivariate logistic regression analyses were performed.
Multivariate logistic regression analysis was conducted using TLR as dependent variable and using age, male gender, obesity (body mass index (BMI), $>30 \mathrm{~kg} / \mathrm{m}^{2}$ ), smoking history, hypertension, dyslipidemia, low-density lipoprotein, diabetes mellitus, glycosylated hemoglobin $\left(\mathrm{HbA}_{\mathrm{cc}}\right)$, characteristics and types of stents, extent of CAD, and UACR as explanatory variables. All statistical analyses were made with Dr. SPSS II release 11.0.1 J (SPSS Japan, Tokyo). A two-tailed value of $P<$ 0.05 was considered statistically significant.

The authors had full access to the statistical data and assume responsibility for its integrity and precision. All authors have read and agreed to the manuscript as written.

\section{RESUltS}

During the study period, 118 patients underwent followup CAG. Of these 118 patients, 103 (79 males and 27 females, mean age, $68.1 \pm 11.9$ years) were negative for qualitative tests for urine protein. During follow-up, 32 of 103 (31.0\%) patients underwent TLR (Figure 1). Patient characteristics at baseline were as follows: BMI, $24.6 \pm 3.6 \mathrm{~kg} / \mathrm{m}^{2}$, hypertension, $57 \mathrm{pa}-$ tients $(55.3 \%)$, dyslipidemia, 70 patients $(68.0 \%)$, diabetes mellitus, 30 patients $(29.1 \%)$, smoking history, 48 patients (46.6\%), and family history of CAD, 26 patients (25.2\%). Single-, two-, and three-vessel lesions were found in 79, 24, and 0 patients, respectively. Late loss occurred in the right coronary artery, left main trunk, left anterior descending artery, and left circumflex artery in 41, 1, 63, and 22 patients, respectively. The left ventricular contraction rate was $58 \pm 11 \%(22-78 \%)$, and each patient had 1.2 lesions. Coronary stents used were $3.04 \pm 0.41 \mathrm{~mm}$ in diameter and $21.7 \pm 8.0 \mathrm{~mm}$ in length. The mean rates of stenosis were $93 \%, 11 \%$, and $41 \%$ before PCI, immediately after PCI, and at the time of follow-up CAG, respectively. MLD was $1.94 \pm 0.86 \mathrm{~mm}$. None of patients required the conduct of follow-up CAG earlier than the scheduled date due to the occurrence of a cardiovascular event. Regarding underlying disease, 50, 42, 3, and 8 patients had acute coronary syndrome, effort angina, old myocardial infarction, and asymptomatic myocardial ischemia, respectively.

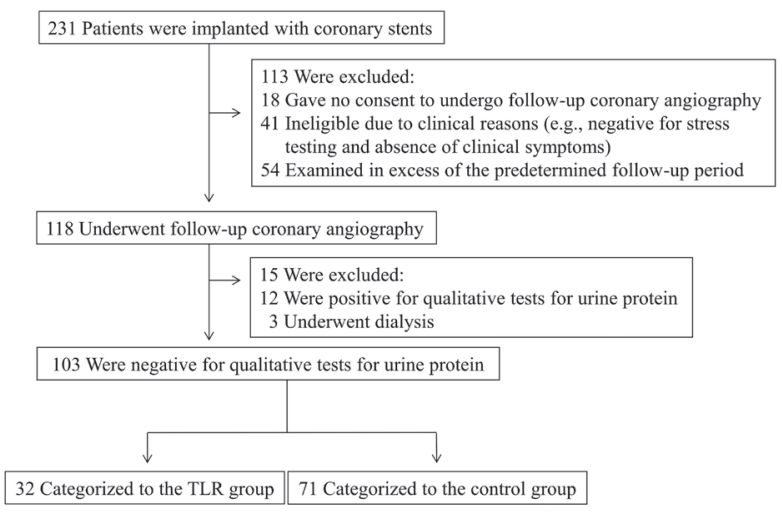

Figure 1. Patient disposition. Of 118 patients who underwent follow-up coronary angiography at 6 to 9 months after percutaneous coronary intervention with coronary stents, 103 negative for qualitative tests for urine protein and not on dialysis were categorized into the TLR group $(n=32)$ and the control group $(n=71)$.CAG indicates coronary angiography; TLR, target lesion revascularization. 
A

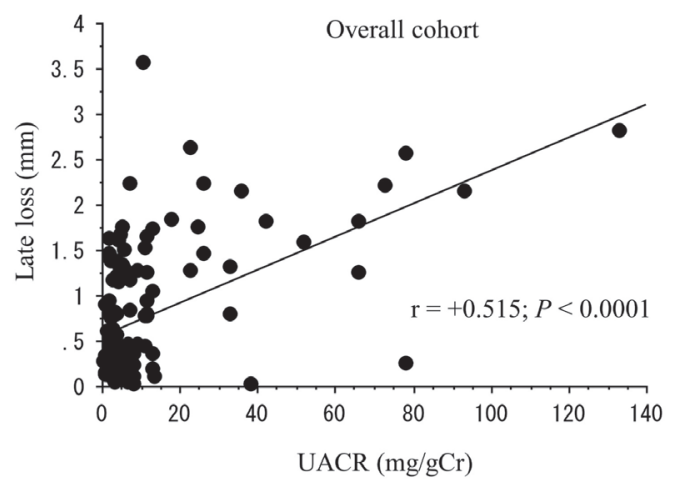

$\mathrm{C}$

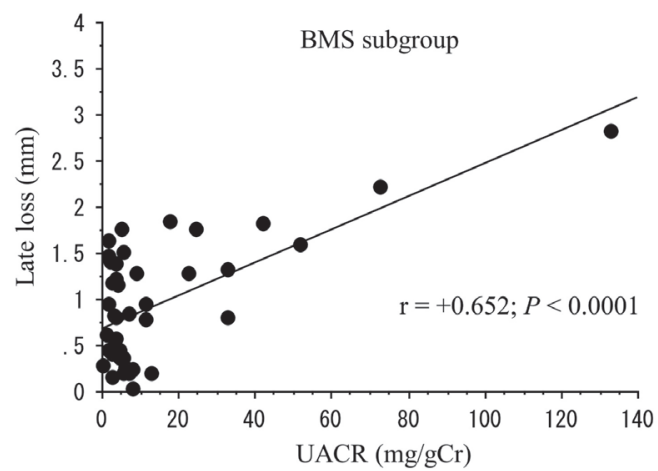

B

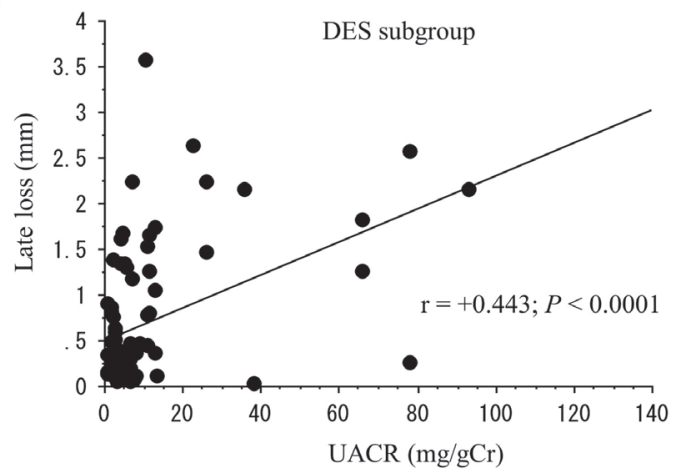

Figure 2. Correlations between UACR and late loss. A: In the overall cohort, a positive correlation $(r=+0.515, P<0.0001)$ was found between UACR and late loss. B: In the DES subgroup, a positive correlation $(r=+0.443, P<0.0001)$ was found between UACR and late loss. C: In the BMS subgroup, a positive correlation $(r=+0.652, P<0.0001)$ was found between UACR and late loss. DES indicates drug-eluting stent; BMS, bare metal stent; and UACR, urinary albumin-to-creatinine ratio.

Table I. Patient Characteristics at the Time of Follow-Up Coronary Artery Angiography

\begin{tabular}{lccc}
\hline Characteristics & $\begin{array}{c}\text { TLR group } \\
(n=32)\end{array}$ & $\begin{array}{c}\text { Control group } \\
(n=71)\end{array}$ & $P$ \\
\hline Age, years & $69.3 \pm 12.3$ & $67.5 \pm 11.8$ & 0.50 \\
Male gender, $n(\%)$ & $21(65.6)$ & $56(78.9)$ & 0.15 \\
Obesity (BMI, $\left.\mathrm{kg} / \mathrm{m}^{2}\right)$ & $24.8 \pm 3.7$ & $24.5 \pm 3.5$ & 0.64 \\
Systolic blood pressure, mmHg & $127 \pm 16$ & $125 \pm 15$ & 0.57 \\
Diastolic blood pressure, mmHg & $72 \pm 7$ & $72 \pm 11$ & 0.80 \\
Hypertension, $n(\%)$ & $21(65.6)$ & $36(50.7)$ & 0.16 \\
Dyslipidemia, $n(\%)$ & $25(78.1)$ & $45(63.4)$ & 0.14 \\
Diabetes mellitus, $n(\%)$ & $10(31.3)$ & $20(28.1)$ & 0.75 \\
Smoking history, $n(\%)$ & $16(50.0)$ & $32(82.1)$ & 0.64 \\
Family history of coronary artery disease, $n(\%)$ & $9(28.1)$ & $17(23.9)$ & 0.65 \\
PCI or CABG, $n(\%)$ & $3(9.4)$ & $6(8.4)$ & 0.57 \\
PAD, $n(\%)$ & $4(12.5)$ & $4(5.6)$ & 0.25 \\
\hline
\end{tabular}

TLR indicates target lesion revascularization; BMI, body mass index; PCI, percutaneous coronary intervention; CABG, coronary artery bypass grafting; and PAD, peripheral arterial disease.

The correlations between UACR and late loss calculated based on MLD measured by QCA are shown in Figure 2. A significantly positive correlation was found between UACR and late loss in the overall cohort (Panel A, $n=128, r=$ $+0.515, P<0.0001)$. Furthermore, significantly positive correlations were also found between UACR and late loss in the DES (Panel B, $n=85, r=+0.443, P<0.0001$ ) and BMS (Panel C, $n=43, r=+0.652, P<0.0001)$ subgroups. In addition, patients who underwent TLR [TLR group] and patients who did not undergo TLR [control group] were analyzed. Conse- quently, no statistically significant difference was found between the two groups with respect to patient characteristics at the time of follow-up CAG as shown in Table I: age, male gender, BMI, systolic blood pressure, diastolic blood pressure, hypertension, dyslipidemia, diabetes mellitus, smoking history, and family history of CAD. Moreover, a statistically significant difference was not found with respect to either pharmacotherapies for patients at the time of follow-up CAG (Table II), or lesion sites, diagnoses, stent diameter, stent length, stent types, and number of lesions at the time of follow-up CAG (Table 
Table II. Pharmacotherapies for Patients at the Time of Follow-Up Coronary Angiography

\begin{tabular}{lccc}
\hline Drugs & $\begin{array}{c}\text { TLR group } \\
(n=32)\end{array}$ & $\begin{array}{c}\text { Control group } \\
(n=71)\end{array}$ & $P$ \\
\hline ACEIs or ARBs, $n(\%)$ & $29(90.6)$ & $57(80.3)$ & 0.19 \\
Antialdosterone drugs, $n(\%)$ & $12(37.5)$ & $18(25.4)$ & 0.21 \\
Beta blockers, $n(\%)$ & $16(50.0)$ & $31(43.7)$ & 0.55 \\
Statins, $n(\%)$ & $22(68.8)$ & $41(57.8)$ & 0.29 \\
Antidiuretics, $n(\%)$ & $5(15.6)$ & $13(18.3)$ & 0.74 \\
Oral antidiabetics, $n(\%)$ & $6(18.7)$ & $16(22.5)$ & 0.75 \\
Insulin, $n(\%)$ & $2(6.3)$ & $2(2.8)$ & 0.40 \\
Urate-lowering agents, $n(\%)$ & $6(18.8)$ & $7(9.9)$ & 0.21 \\
Aspirin, $n(\%)$ & $32(100.0)$ & $71(100.0)$ & 1.00 \\
Antiplatelets, $n(\%)$ & & & 0.12 \\
$\quad$ Clopidogrel & $25(78.1)$ & $58(81.7)$ & $11(15.5)$ \\
Ticlopidine & $3(9.4)$ & $2(2.8)$ & \\
Cilostazol & $4(12.5)$ & \\
\hline
\end{tabular}

TLR indicates target lesion revascularization; ACEIs, angiotensin-converting enzyme inhibitors; and ARBs, angiotensin II receptor blockers.

Table III. Lesion Characteristics, Diagnoses, and Stent Profiles at the Time of Follow-Up Coronary Angiography

\begin{tabular}{|c|c|c|c|}
\hline Variables & $\begin{array}{l}\text { TLR group } \\
\quad(n=32)\end{array}$ & $\begin{array}{l}\text { Control group } \\
\quad(n=71)\end{array}$ & $P$ \\
\hline Lesion sites, $n(\%)$ & & & 0.37 \\
\hline $\mathrm{RCA}$ & $13(40.6)$ & $23(32.4)$ & \\
\hline LMT & $1(3.1)$ & $0(0.0)$ & \\
\hline LAD & $15(46.8)$ & $39(54.9)$ & \\
\hline LCX & $3(9.4)$ & $9(12.7)$ & \\
\hline Diagnoses, $n(\%)$ & & & 0.64 \\
\hline Acute coronary syndrome & $16(50.0)$ & $34(47.9)$ & \\
\hline Effort angina & $11(34.4)$ & $31(43.7)$ & \\
\hline Old myocardial infarction & $2(6.3)$ & $1(1.4)$ & \\
\hline Asymptomatic myocardial ischemia & $3(9.4)$ & $5(7.0)$ & \\
\hline Stent diameter, $\mathrm{mm}$ & $3.09 \pm 0.37$ & $3.01 \pm 0.42$ & 0.41 \\
\hline Stent length, mm & $20.1 \pm 6.4$ & $22.4 \pm 8.5$ & 0.17 \\
\hline Stent types, $n(\%)$ & & & 0.49 \\
\hline Drug-eluting stent & $18(56.3)$ & $45(63.4)$ & \\
\hline Bare metal stent & $14(43.8)$ & $26(36.6)$ & \\
\hline Types of lesions, $n(\%)$ & & & $<0.05$ \\
\hline Single vessel lesion & $20(62.5)$ & $59(83.1)$ & \\
\hline Multivessel lesion & $12(37.5)$ & $12(16.9)$ & \\
\hline
\end{tabular}

TLR indicates target lesion revascularization; RCA, right coronary artery; LMT, left main trunk; LAD, left anterior descending artery; and LCX, left circumflex artery.

III). With a single vessel lesion as SVL and two vessel lesions as multiple vessel lesions (MVL), the incidence of MVL was significantly higher $(P<0.05)$ in the TLR group than in the control group (Table III). No significant difference was found between the two groups with respect to hematology and blood chemistry (Table IV), while UACR was significantly higher $(P$ $<0.01)$ in the TLR group $(23.88 \pm 31.80 \mathrm{mg} / \mathrm{gCr})$ than in the control group $(6.29 \pm 7.46 \mathrm{mg} / \mathrm{gCr})$.

The results from univariate and multivariate logistic regression analyses of variables associated with TLR after PCI are shown in Table V. Univariate analysis revealed no significant difference with respect to all examined variables except MVL [odds ratio: 2.95 ; $95 \%$ confidence interval (CI): 1.14 7.61; $P=0.02$ ) and UACR (odds ratio: 1.07 ; 95\% CI: $1.02-$ $1.12 ; P<0.01)]$. Multivariate analysis revealed that MVL (odds ratio: 3.03 ; $95 \% \mathrm{CI}: 1.08-8.53 ; P=0.04$ ) and UACR (odds ratio: $1.07,95 \% \mathrm{CI}$ : 1.02-1.12; $P<0.01$ ) were independent predictors of TLR and that UACR was a significant predic- tor of TLR in SVL (odds ratio: 1.05; 95\% CI: 1.01-1.10; $P=$ 0.02 ), but not in MVL (odds ratio: 1.37 ; $95 \%$ CI: 0.97-1.92; $P$ $=0.07)$.

\section{Discussion}

UACR is generally used as a marker for diabetic nephropathy and has also been reported as a risk factor for arteriosclerosis in many studies. ${ }^{1-7)}$ Therefore, UACR is a potential marker for arteriosclerosis. Microalbuminuria caused by glomerular damage seems to be associated with endothelial injury of the glomeruli, which led us to consider that UACR can be used as a variable for arteriosclerotic changes in CAD as well.

Multivariate logistic regression analysis revealed a significantly higher $(P<0.01)$ UACR in the TLR group than in the control group with respect to TLR after PCI, and a positive as- 
Table IV. Hematology, Blood Chemistry, and Urinalysis at the Time of Follow-Up Coronary Angiography

\begin{tabular}{|c|c|c|c|}
\hline Variables & $\begin{array}{l}\text { TLR group } \\
\quad(n=32)\end{array}$ & $\begin{array}{l}\text { Control group } \\
\quad(n=71)\end{array}$ & $P$ \\
\hline White blood cell count, $\times 10^{3} / \mu \mathrm{L}$ & $5.76 \pm 1.23$ & $5.46 \pm 1.35$ & 0.29 \\
\hline Red blood cell count, $\times 10^{4} / \mu \mathrm{L}$ & $434 \pm 69$ & $427 \pm 50$ & 0.54 \\
\hline Hemoglobin, g/dL & $12.7 \pm 2.0$ & $12.8 \pm 1.3$ & 0.55 \\
\hline Platelet count, $\times 10^{4} / \mu \mathrm{L}$ & $21.2 \pm 4.3$ & $20.1 \pm 4.5$ & 0.26 \\
\hline Urea nitrogen, mg/dL & $15.2 \pm 3.9$ & $15.8 \pm 3.7$ & 0.47 \\
\hline Creatinine, $\mathrm{mg} / \mathrm{dL}$ & $0.81 \pm 0.18$ & $0.89 \pm 0.19$ & 0.06 \\
\hline AST, IU/L & $21.2 \pm 6.5$ & $19.2 \pm 4.1$ & 0.06 \\
\hline ALT, IU/L & $20.0 \pm 11.7$ & $17.0 \pm 6.2$ & 0.09 \\
\hline LDH, IU/L & $174.7 \pm 17.5$ & $163.7 \pm 33.2$ & 0.08 \\
\hline Triglycerides, mg/dL & $131.3 \pm 88.0$ & $135.9 \pm 80.0$ & 0.79 \\
\hline HDL-C, mg/dL & $51.7 \pm 14.5$ & $50.3 \pm 14.7$ & 0.65 \\
\hline LDL-C, mg/dL & $94.6 \pm 28.8$ & $89.9 \pm 19.8$ & 0.34 \\
\hline LDL/HDL-C ratio & $2.05 \pm 1.05$ & $1.95 \pm 0.71$ & 0.54 \\
\hline $\mathrm{HbA}_{1 \mathrm{c}}, \%$ & $5.92 \pm 1.17$ & $5.59 \pm 0.72$ & 0.08 \\
\hline Uric acid, mg/dL & $5.99 \pm 2.17$ & $6.02 \pm 1.42$ & 0.93 \\
\hline $\mathrm{CRP}, \mathrm{mg} / \mathrm{dL}$ & $0.24 \pm 0.35$ & $0.18 \pm 0.30$ & 0.39 \\
\hline $\mathrm{UACR}, \mathrm{mg} / \mathrm{gCr}$ & $23.88 \pm 31.8$ & $6.29 \pm 7.46$ & $<0.01$ \\
\hline
\end{tabular}

TLR indicates target lesion revascularization; AST, aspartate aminotransferase; ALT, alanine aminotransferase; LDH, lactate dehydrogenase; HDL-C, high-density lipoprotein cholesterol; LDL-C, low-density lipoprotein cholesterol; HbA $1 \mathrm{c}$, glycosylated hemoglobin; CRP, C-reactive protein; and UACR, urinary albumin-to-creatinine ratio.

Table V. Univariate and Multivariate Logistic Regression Analyses of Variables Associated With Target Lesion Revascularization After Percutaneous Coronary Intervention With Coronary Stents

\begin{tabular}{|c|c|c|c|c|}
\hline Explanatory variables & $\begin{array}{c}\text { Univariate analysis } \\
\text { OR }(95 \% \mathrm{CI})\end{array}$ & $P$ & $\begin{array}{c}\text { Multivariate analysis } \\
\text { OR }(95 \% \mathrm{CI})\end{array}$ & $P$ \\
\hline Age, years & $1.01(0.98-1.05)$ & 0.49 & - & - \\
\hline Male gender & $0.51(0.20-1.29)$ & 0.16 & - & - \\
\hline Obesity (body mass index, $>30 \mathrm{~kg} / \mathrm{m}^{2}$ ) & $1.73(0.36-8.24)$ & 0.49 & - & - \\
\hline Smoking history & $1.22(0.53-2.81)$ & 0.64 & - & - \\
\hline Hypertension & $1.86(0.78-4.41)$ & 0.16 & - & - \\
\hline Dyslipidemia & $2.06(0.78-5.43)$ & 0.14 & - & - \\
\hline LDL-C & $1.01(0.99-1.03)$ & 0.34 & - & - \\
\hline Diabetes mellitus & $1.16(0.47-2.88)$ & 0.75 & - & - \\
\hline $\mathrm{HbA}_{1 \mathrm{c}}$ & $1.48(0.92-2.39)$ & 0.08 & - & - \\
\hline \multicolumn{5}{|l|}{ Characteristics and types of stents } \\
\hline Stent diameter & $1.54(0.56-4.26)$ & 0.41 & - & - \\
\hline Stent length & $0.96(0.90-1.02)$ & 0.17 & - & - \\
\hline DES & $0.74(0.32-1.74)$ & 0.49 & - & - \\
\hline \multicolumn{5}{|l|}{ Types of lesions } \\
\hline SVL & $0.34(0.13-0.87)$ & 0.02 & - & - \\
\hline MVL & $2.95(1.14-7.61)$ & 0.03 & $3.03(1.08-8.53)$ & 0.04 \\
\hline PCI or CABG & $1.12(0.26-4.79)$ & 0.57 & - & - \\
\hline PAD & $2.39(0.56-10.25)$ & 0.25 & - & - \\
\hline UACR & $1.07(1.02-1.12)$ & $<0.01$ & $1.07(1.02-1.12)$ & $<0.01$ \\
\hline
\end{tabular}

OR indicates odds ratio; CI, confidence interval; DES, drug-eluting stent; BMS, bare metal stent; CAD, coronary artery disease; SVL, single vessel lesion; MVL, multivessel lesion; LDL-C, low-density lipoprotein cholesterol; $\mathrm{HbA}_{1 \mathrm{c}}$, glycosylated hemoglobin; and UACR, urinary albumin-to-creatinine ratio.

sociation $(r=+0.515, P<0.0001)$ was found between UACR and late loss. Therefore, the present study indicates that UACR is a potential predictor of TLR required for severe ISR after PCI with coronary stents. Furthermore, elevated UACR appears to be associated with arteriosclerosis progression because of being its predictor. The results from the present study support the hypothesis that arteriosclerotic changes (eg, endothelial damage), which are associated with glomerular damage, may lead to stent malexpansion and/or malapposition and induce stent restenosis. We conjectured that elevated UACR may be a risk factor for ISR after PCI in patients with advanced ar- teriosclerotic disease. However, clinical studies on ISR after coronary revascularization conducted by Toplak, et al. and Sukhija, et al. indicated no significant difference in microalbuminuria. ${ }^{12,13)}$ This discrepancy may be explained at least in part by the following facts: low-density lipoprotein (LDL) cholesterol was well controlled in our patients compared to their patients; and aggressive therapy to lower LDL cholesterol possibly reduced the incidence of ISR, which successfully demonstrated a significant difference in UACR. Furthermore, Sukhija, et al $^{13)}$ showed no significant difference in microalbuminuria probably because 1) they used conventional cutoff val- 
ues for microalbuminuria and 2) they included patients with moderate ISR in contrast to our study in which we included patients with severe ISR only. We speculate that UACR showed a significant difference in the degree of association with TLR after PCI with BMSs and/or DESs, probably due to the following facts: 1) the study population was placed under the strict control of risk factors for arteriosclerosis; 2) there was no significant difference in laboratory data (eg, LDL and $\mathrm{HbA}_{1 \mathrm{c}}$ ) between the TLR group and the control group, probably because of small sample size; and 3) the ultratrace amount of albumin in urine was assayed using UACR as a variable for microalbuminuria. Furthermore, the mean UACR value in the TLR group was $23.88 \mathrm{mg} / \mathrm{gCr}$-an undetectable concentration in qualitative tests for urine protein. Lower threshold levels for UACR need to be established when used as a risk factor for $\mathrm{CVD},{ }^{14)}$ and the results from the present study also support the review of threshold levels. However, UACR was found to be a significant predictor of TLR in SVL, but not in MVL. We consider that this fact was probably attributed to a small subpopulation of MVL resulting from the subpopulation formation, implying a need to conduct another clinical study including the greater subpopulation of MVL.

Furthermore, our study indicated a definite correlation between UACR and late loss. We believe that arteriosclerosis progression, which appears to be indicated by elevated UACR, is involved at least partly in the generation of late loss that may eventually provoke severe ISR. The fact that DESs had a lower correlation coefficient between UACR and late loss than did BMSs leads us to speculate that the neointimal proliferationinhibiting effect of DESs suppressed severe late loss. Nevertheless, we did not calculate UACR during PCI. The present study hence included a number of patients who underwent medical interventions, making it unclear whether elevated UACR at the time of follow-up CAG after PCI was caused by arteriosclerosis progression after PCI or by arteriosclerosis that had progressed already at the time of PCI. In addition, the possible effects of angiotensin-converting enzyme inhibitors, angiotensin II receptor blockers, and statins that lower UACR ${ }^{15-24)}$ cannot be ruled out in the present study. We believe that the calculation of UACR during PCI and at the time of follow-up CAG in a future study will allow us to examine the effects of medical interventions after PCI.

No significant differences were found in other predictors of ISR, eg, LDL cholesterol, LDL/high-density lipoprotein ratio, $\mathrm{HbA}_{1 \mathrm{c}}$, stent diameter, and stent length, which may be attributable to the facts that the patients enrolled were placed under the strict control of risk factors for arteriosclerosis and that the sample size was small. Among these variables, UACR showed a markedly significant difference $(P<0.0001)$ and implied its nature of a strong predictor of TLR. However, MVL had a 2.75 -fold greater odds ratio and a $>4$-fold less strength of evidence as expressed by the $P$-value compared to UACR. In the present study, the number of patients with MVL was significantly greater $(P<0.05)$ in the TLR group than in the control group. Vacek, et $\mathrm{al}^{25)}$ found no significant difference in ISR between patients with MVL and patients with SVL. In contrast, Geluk, et $a l^{26)}$ reported a greater incidence of ISR in patients with MVL than in patients with SVL. The former group of investigators examined patients with acute myocardial infarction only, while the latter investigated patients with stable angina pectoris, patients with unstable angina pectoris, and patients with old myocardial infarction. A greater similarity in study design is noted between ours and the latter study, and our study results support theirs.

ISR is a serious complication of stent implantation that still develops in clinical settings, although its incidence was reduced by the introduction of stent devices of new generations and by the strict control of risk factors for ISR. Our study indicating UACR as a potential predictor of TLR suggests that early therapeutic intervention as secondary prevention of severe ISR by lowering UACR possibly further reduces the incidence of the stent-derived complication.

Our patients showed an ISR rate as high as $30.2 \%$ but had no clinical symptoms. We performed exercise stress tests in patients who did not provide written consent to undergo follow-up CAG. Hence, we followed them up clinically without conducting CAG when the test result was negative. We presume that this less aggressive clinical approach was translated into such an elevated incidence of ISR after PCI.

Study limitations: The present study has several limitations. First, it is retrospective in design, preoperative stenting in patients was not blinded, and sample size was small, which cannot rule out a number of biases (eg, selection bias, treatment bias, and information bias). Second, late loss was assessed by QCA not by intravenous ultrasound (IVUS). Although IVUS is ideally preferred to assess late loss, we believe it is difficult to perform IVUS in all patients who do not present with ISR during following-up after PCI in clinical settings. An open-label prospective clinical study to investigate a greater number of patients with CAD in the target population will validate UACR as a predictor of ISR after PCI.

Conclusions: Our study demonstrates that 1 ) there were positive associations between UACR and late loss-a variable for the assessment of TLR-in the overall cohort, the DES subgroup, and the BMS subgroup, 2) UACR, which can be easily calculated based on values measured in routine, noninvasive, inexpensive urinalysis, is a potential predictor of TLR that is required for severe ISR after PCI in stent-implanted patients, and 3) UACR might indirectly predict severe ISR and could serve as an index for its secondary prevention. Further clinical research is warranted to validate the clinical relevance of our observations.

\section{ACKNOWLEDGMENTS}

The authors thank Satoshi Sakima, MD, for his valuable suggestions and critical review of the manuscript.

\section{ReFERENCES}

1. Levey AS, Eckardt KU, Tsukamoto Y, et al. Definition and classification of chronic kidney disease: a position statement from Kidney Disease: Improving Global Outcomes (KDIGO). Kidney Int 2005; 67: 2089-100.

2. Ruilope LM, Bakris GL. Renal function and target organ damage in hypertension. Eur Heart J 2011; 32: 1599-604. (Review)

3. Hillege HL, Fidler V, Diercks GF, et al; Prevention of Renal and Vascular End Stage Disease (PREVEND) Study Group. Urinary albumin excretion predicts cardiovascular and noncardiovascular mortality in general population. Circulation 2002; 106: 1777-82.

4. Rein P, Vonbank A, Saely $\mathrm{CH}$, et al. Relation of albuminuria to 
angiographically determined coronary arterial narrowing in patients with and without type 2 diabetes mellitus and stable or suspected coronary artery disease. Am J Cadriol 2011; 107: 1144-8.

5. Son JW, Jang EH, Kim MK, et al. Usefulness of albuminuria as predictor for coronary artery stenosis, regardless of estimated glomerular filtration rate, in patients with type 2 diabetes mellitus. Am J Cardiol 2012; 110: 1434-9.

6. Klausen KP, Scharling H, Jensen JS. Very low level of microalbuminuria is associated with increased risk of death in subjects with cardiovascular or cerebrovascular diseases. J Intern Med 2006; 260: 231-7.

7. Wang J, Ruotsalainen S, Moilanen L, Lepistö P, Laakso M, Kuusisto J. The metabolic syndrome predicts cardiovascular mortality: a 13-year follow-up study in elderly non-diabetic Finns. Eur Heart J 2007; 28: 857-64.

8. Diercks GF, van Boven AJ, Hillege HL, et al. Microalbuminuria is independently associated with ischaemic electrocardiographic abnormalities in a large non-diabetic population. The PREVEND (Prevention of Renal and Vascular ENdstage Disease) study. Eur Heart J 2000; 21: 1922-7.

9. Arnlöv J, Evans JC, Meigs JB, et al. Low-grade albuminuria and incidence of cardiovascular disease events in nonhypertensive and nondiabetic individuals: the Framingham Heart Study. Circulation 2005; 112: 969-75.

10. Ryan TJ, Faxon DP, Gunnar RM, et al. Guidelines for percutaneous transluminal angioplasty. A report of the American College of Cardiology/American Heart Association Task Force on Assessment of Diagnostic and Therapeutic Cardiovascular Procedures (Subcommittee on Percutaneous Transluminal Coronary Angioplasty). Circulation 1988; 78: 486-502.

11. Teppo AM. Immunoturbidimetry of albumin and immunoglobulin $\mathrm{G}$ in urine. Clin Chem 1982; 28: 1359-61.

12. Toplak H, Schumacher M, Eber B, Luha O, Klein W, Krejs GJ. Microalbuminuria is no risk restenosis following percutaneous transluminal coronary angioplasty. Clinical Investig 1992; 70: 1010-2.

13. Sukhija R, Aronow WS, Sureddi R, et al. Predictors of in-stent restenosis and patient outcome after percutaneous coronary intervention in patients with diabetes mellitus. Am J Cradiol 2007; 100: 777-80.

14. Ruggenenti P, Remuzzi G. Time to abandon microalbuminuria? Kidney Int 2006; 70: 1214-22. (Review)

15. Ravid M, Savin H, Jutrin I, Bental T, Katz B, Lishner M. Longterm stabilizing effect of angiotensin-converting enzyme inhibition on plasma creatinine and on proteinuria in normotensive type II diabetic patients. Ann Intern Med 1993; 118: 577-81.

16. Muirhead N, Feagan BF, Mahon J, et al. The effects of valsartan and captopril on reducing microalbuminuria in patients with type 2 diabetes mellitus: a placebo-controlled trial. Curr Therapeutic Res 1999; 60: 650-60.

17. Viberti G, Wheeldon NM; MicroAlbuminuria Reduction With VALsartan (MARVAL) Study Investigators. Microalbuminuria reduction with valsartan in patients with type 2 diabetes mellitus: A blood pressure-independent effect. Circulation 2002; 106: 672-8.

18. The GISEN Group(Gruppo Italiano di Studi Epidemiologici in Nefrologia). Randomised placebo-controlled trial of effect of ramipril on decline in glomerular filtration rate and risk of terminal renal failure in proteinuric, non-diabetic nephropathy. Lancet 1997; 349: 1857-63.

19. Parving HH, Lehnert H, Bröchner-Mortensen J, et al; Irbesartan in Patients with Type 2 Diabetes and Microalbuminuria Study Group. The effect of irbesartan on the development of diabetic nephropathy in patients with type 2 diabetes. N Engl J Med 2001; $345: 870$ 8 .

20. Asselbergs FW, Diercks GF, Hillege HL, et al; Prevention of Renal and Vascular Endstage Disease Intervention Trial (PREVEND IT) Investigators. Effects of fosinopril and pravastatin on cardiovascular events in subjects with microalbuminuria. Circulation 2004; 110: 2809-16.

21. Velussi M, Cernigoi AM, Tortul C, Merni M. Atorvastatin for the management of Type 2 diabetic patients with dyslipidaemia. A mid-term ( 9 months) treatment experience. Diabetes Nutr Metab 1999; 12: 407-12.

22. Velussi M. Long-term (18-month) efficacy of atorvastatin therapy on type 2 diabetics at cardiovascular risk. Nutr Metab Cardiovasc Dis 2002; 12: 29-35.

23. Sinzinger H, Kritz H, Furberg CD. Atorvastatin reduces microalbuminuria in patients with familial hypercholesterolemia and normal glucose tolerance. Med Sci Monit 2003; 9: P188-92.

24. Sandhu S, Wiebe N, Fried LF, Tonelli M. Statins for improving renal outcomes: a meta-analysis. J Am Soc Nephrol 2006; 17: 200616. (Review)

25. Vacek JL, Rosamond TL, Robuck W, Kramer PH, Beauchamp GD. Prognosis of culprit lesion PTCA in acute myocardial infarction for multi versus single vessel disease. Cathet Cardiovas Diagn 1991; 24: 161-5.

26. Gürlek A, Dağalp Z, Oral D, et al. Restenosis after transluminal coronary angioplasty: a risk factor analysis. J Cardiovasc Risk 1995; 2 : 51-5. 